\title{
Ruta del Vino Ribeira Sacra (Galicia - España): Análisis desde el punto de vista de la oferta enoturística
}

\author{
Ribeira Sacra Wine Route (Galicia - Spain): Analysis from the wine tourism \\ offer's point of view
}

\author{
María de la Cruz del Río Rama (RIO RAMA, M. de la C. del)* \\ José Álvarez García (ALVAREZ GARCÍA, J.) \\ José Manoel Gonçalves Gândara (GÂNDARA, J. M. G.) ${ }^{* * * *}$ \\ Vander Valduga (VALDUGA, V.) \\ Pilar Rodríguez González (RODRÍGUEZ GONZÁLEZ, P.) ${ }^{* * * * *}$
}

\begin{abstract}
RESUMEN - En España existe una gran tradición vitivinícola que ha dado paso a la creación de las Rutas del Vino, proyecto impulsado por Secretaría General de Turismo y la Asociación Española de Ciudades del Vino (ACEVIN), que se plasmó en el marco del Programa de Calidad en los Productos Turísticos del Plan Integral de Calidad del Turismo - PICTE 2000-2006. En este contexto, se exponen los resultados de un estudio empírico llevado a cabo en las bodegas, restaurantes y alojamientos hoteleros adheridos a la Ruta del Vino del Ribeira Sacra en el año 2013, con el objetivo de analizar la ruta desde el punto de vista de la oferta enoturística. La metodología consistió en un análisis descriptivo llevado a cabo en 27 establecimientos de un total de 35 que conforman la ruta. Los resultados obtenidos permitirán dar respuesta a todos los objetivos planteados, indicando el 38,46\% de los establecimientos adheridos un nivel alto de satisfacción con su adhesión frente al 11,54\% que no están satisfechos y solo el 18,52\% valoran la actividad enoturística de la ruta como buena o muy buena, manifestando el 51,85\% de los establecimiento una valoración neutra (ni buena, ni mala).
\end{abstract}

Palabras clave: Enoturismo; Ruta del Ribeira Sacra; Oferta; Galicia (España).

*PhD. Dirección de Empresas (Universidad de Vigo, España), Profesora Contratada Doctora en la Universidad de Vigo (Campus Ourense). Facultad de Ciencias Empresariales y Turismo. As Lagoas, s./n. 32004 Ourense, España. Teléfono: 34 649667878. E-mail: delrio@uvigo.es

**PhD. Dirección y Planificación de Turismo (Universidad de Vigo, España), Profesor Ayudante Doctor en la Universidad de Extremadura (Campus Cáceres), Facultad de Estudios Empresariales y Turismo, Departamento de Economía Financiera y Contabilidad. Avenida de la Universidad, n. 47, 10003 Cáceres (España). Teléfono: 34 609880141. E-mail: pepealvarez@uvigo.es

***Phd. Turismo y Desarrollo Sostenible (Universidad de Las Palmas de Gran Canaria, España). Profesor Adjunto del Departamento de Turismo de la Universidad Federal de Paraná (Brasil). Coordinador del Máster en Turismo de la Universidad Federal de Paraná (Brasil). Calle Dr. Faivre, 405 (3er piso), Curitiba, Paraná, Brasil. Teléfono: 41 3360-5050. E-mail: jmggandar@yahoo.com.br

****Phd. Geografía. (Universidad Federal de Río Grande do Sul, Brasil). Profesor Adjunto del Departamento de Turismo de la Universidad Federal de Paraná (Brasil) y del Máster en Turismo de la Universidad Federal de Paraná (Brasil). Calle Dr. Faivre, 405 (3er piso), Curitiba, Paraná, Brasil. Teléfono: 41 3360-5050. E-mail: E-mail: vandervalduga@gmail.com

*****Máster Universitario en Dirección y Planificación del Turismo de Interior y de Salud (Universidad de Vigo, España). Facultad de Ciencias Empresariales y Turismo. As Lagoas, s./n. 32004 Ourense. Teléfono: 34 988368727. Email: pilar_rodriguezgonzalez@hotmail.com 
ABSTRACT - Spain has an important and historical viticulture tradition that has led to the creation of the Wine Routes. This project was encouraged by the General Tourism Secretary and the Association of Wine Cities of Spain (ACEVIN), which was structured within the "Quality Program of Tourist Products" of the "Integral Plan for Tourism Quality- PICTE 2000-2006". In this context, results of an empiric study carried out in the wineries, restaurants and hotels adhered to Ribera Sacra Wine Route in 2013 are exposed, with the aim to analyze it under the perspective of the wine tourist offer. The methodology consisted in a descriptive analysis conducted in 27 establishments of a total of 35 that comprise the wine route. The obtained results will permit to answer to all the proposed questions, indicating that the $38,46 \%$ of the adhered establishments are very satisfied with their adhesion, face to the $11,54 \%$ that are not satisfied and only $18,52 \%$ qualified the Wine Route as good or very good, while $51,85 \%$ of the total are neutral and they don't consider it neither good nor bad.

Key words: Wine Tourism; Ribeira Sacra Wine Route; Tourist offer; Galicia (Spain). 


\section{INTRODUCCIÓN}

La unión de dos actividades económicas tan dispares como son el turismo y la viticultura dan lugar al nacimiento de un nuevo concepto que es el "enoturismo" que se encuadra dentro del denominado turismo temático, donde estas dos actividades se acoplan para crear una actividad conjunta (ANDREU; VERDÚ, 2012, p. 38). El “enoturismo" es definido por la Carta Europea de Enoturismo (RECEVIN, 2006, p. 2) como "el desarrollo de las actividades turísticas, de ocio y tiempo libre, dedicadas al descubrimiento y disfrute cultural y enológico de la viña, el vino y su territorio".

Aunque el enoturismo es una actividad de reciente implantación en España, cada día coge un mayor empuje. Este nuevo producto turístico surge en España debido a dos factores, por un lado, para dar respuesta al cambio en las necesidades y nuevos gustos de los clientes, que en la actualidad tienden a repartir el periodo vacacional en varios períodos a lo largo del año, realizando pequeñas “escapadas” (RODRÍGUEZ GARCIA et al., 2010) lo cual hace que un destino enoturístico resulte interesante para un mayor número de visitantes sin necesidad de que sean expertos en el mundo del vino. Y por otro, los empresarios de las empresas vitivinícolas ven en el enoturísmo una oportunidad para diversificar su producto o un nuevo posicionamiento estratégico que los situé en un nicho de mercado sin explotar (MEDINA; TRESSERRAS, 2008, p. 494).

Así, como respuesta a la demanda enoturística en España surge el producto "Ruta del Vino" definida por Bruwer (2003, p. 424) como "una ruta turística que une varias bodegas y viñedos en una zona determinada" y ACEVIN (2009a, p. 17) como:

[...] la integración en un único producto de recursos y servicios turísticos de interés, existentes y potenciales, de una zona vitivinícola. Busca construir una identidad propia del destino que facilite la comercialización y garantice la satisfacción de la demanda, impulsando el desarrollo económico y social.

El turismo enológico se desarrolla habitualmente en el ámbito rural permitiendo generar puestos de trabajo en zonas, que por su carácter geográfico y su actividad centrada en el sector primario, tienen más dificultades para hacerlo, y de esta manera, evitar la despoblación de la zona, al mismo tiempo que genera desarrollo económicosocial (SIMÕES, 2008, p. 273-274). Las rutas del vino se crean con la finalidad de 
llevar a cabo uno o varios objetivos (HALL, MICHELL, 2001, p. 448-449; LÓPEZGUZMÁN; SÁNCHEZ CAÑIZARES, 2008, p. 164-166; RAMIS, 2010, p. 25; ALPÍZAR; MALDONADO, 2011, p. 97-110): (1) Generar riqueza y empleo en zonas rurales, (2) Contribuir a financiar el coste de las diferentes infraestructuras, (3) Desarrollar otras actividades económicas, (4) Crear una oferta lúdica para la comunidad local (por ejemplo, actividades recreativas) y un conjunto de servicios, (5) Conservar los recursos medioambientales y culturales, (6) Reforzar la identidad e imagen que deriva de la Denominación de Origen, (7) Facilitar el acceso a nuevos mercados, (8) Desestacionalizar la demanda.

En este contexto, y dada la importancia de los objetivos a conseguir por toda ruta y su impacto social y económico en la zona se identificó de gran relevancia la investigación en este ámbito, estudios que hasta el momento son muy escasos (DEL RÍO et al., 2013 y DEL RÍO; ÁLVAREZ; FRAIZ, 2014. Por todo ello, el objetivo principal de esta investigación fue realizar un análisis de la Ruta del Vino Ribeira Sacra desde el punto de vista de su oferta enoturística (bodegas, restaurantes y alojamientos hoteleros) adheridos a la ruta.

Como objetivos parciales se han definidos comprender el perfil de los establecimientos que forman parte de la ruta, el perfil de los visitantes, las actividades enoturísticas que los establecimientos ofrecen, conocer la satisfacción de los establecimientos adheridos y la valoración global de la actividad enoturística en la ruta, y para finalizar realizar un análisis (Fortalezas/Oportunidades/Debilidades/Amenazas) de la Ruta del Vino Ribeira Sacra con la opinión aportada por los gestores de los establecimientos de la Ruta. La zona a estudiar es una de las zonas más importantes de producción de caldos en Galicia, la "Ribeira Sacra", tierras que se extienden principalmente a lo largo de las riberas de los ríos Miño y, su afluente, el Sil, situadas entre el Sur de la provincia de Lugo y Norte de la provincia de Ourense.

Para poder dar respuesta a los objetivos planteados el trabajo se ha estructurado en varios apartados. En la siguiente sección se define lo que es una ruta turística enológica y se describe la Ruta Ribeira Sacra, ruta en la que se llevo a cabo el proyecto de investigación que se presenta en este paper. En la sección 3 se detalla la metodología 
de investigación y se presentan los resultados en la sección 4. Finalmente, en la sección 5 se recogen las principales conclusiones obtenidas en la investigación.

\section{RUTAS TURÍSTICAS ENOLÓGICAS}

En el marco del Sistema de Calidad Turístico Español ${ }^{1}$ (SCTE) Destinos se impulsan y crean los Clubs de Producto que se definen como un conjunto de empresas de tamaño pequeño y mediano, las cuales realizan el trabajo de manera coordinada para elaborar nuevos productos y aumentar el valor de los que ya existen. En este contexto, nace el Club de Producto Rutas del Vino de España, estando su origen orientado al "desarrollo local a partir de la puesta en valor del territorio vitivinícola y la potenciación del sector vitivinícola como motor de otras actividades económicas" (ACEVIN, 2012b, p. 2).

Para llevar a cabo el proyecto Rutas del Vino, que nace en el año 2001, se constituyó en 1994, la Asociación Española de Rutas del Vino (ACEVIN) como ente gestor que junto con el apoyo de la Secretaría General de Turismo inicia la definición y desarrollo de las normas de regulación de la calidad de este producto turístico (MILLÁ; DANCAUSA, 2012) dentro del PICTE - Plan Integral de la Calidad del Turismo Español 2000-2006 (PICTE, 1999). Así, las Rutas de Vino surgen dentro del programa "Calidad en los productos Turísticos" que es uno de los diez Programas que se crean en el PICTE, cuyo objetivo es el de trabajar en el diseño de la oferta de productos turísticos, y en su creación y desarrollo, con la finalidad de diversificar y desestacionalizar la actividad turística en España (PICTE, 1999).

Son muchas las definiciones sobre lo que es una Ruta del Vino y todas ellas aportan enfoques diferentes de este producto turístico. Según Miranda Escolar y Fernández Morueco (2011, p. 148-149) una Ruta del Vino:

\footnotetext{
${ }^{1}$ El Sistema de Calidad Turística Española (SCTE) nace en 1996 siendo la promotora La Secretaria General de Turismo, y como Ente Gestor el Instituto de Calidad Turístico Español (ICTE), con el objetivo de facilitar a las empresas de turismo herramientas que les permitan mejorar su posición frente a empresas de la competencia. Los pilares de SCTE son: SCTE Buenas Prácticas, SCTE Destinos y SCTE Sectores (FORONDA; LÓPEZ, 2009).
} 
[...] es un producto turístico basado en la integración de actividades vitícolas, gastronómicas, culturales y de ocio, las cuales se complementan con una amplia oferta de restauración y de alojamiento, servicios relacionados con dicha actividad y otros complementarios, dentro de una misma zona vitivinícola.

Pero sin lugar a dudas "la ruta turística enológica, es el resultado del esfuerzo y la sinergia que derivan de la colaboración entre empresas públicas y privadas, a partir de una alianza y objetivos comunes" (TELFER, 2001, p. 21), que une "tanto a empresas específicamente turísticas, como a otras cuyos sectores han estado tradicionalmente lejos del turismo (bodegas, enotecas, etc.)" (RUTAS DEL VINO DE ESPAÑA, 2012). Pero no se debe olvidar que:

[...] una Ruta del Vino es una herramienta para el desarrollo rural, centrado en un modelo de desarrollo económico sostenible e integrador de determinadas áreas, es capaz de dinamizar la competitividad de un territorio, incrementar y mejorar la producción vinícola, respetar el medio ambiente y mejorar las condiciones de vida de los ciudadanos" (MILLÁN; MELIÁN, 2008, p. 162).

Como ya se ha mencionado, las Rutas del Vino se crean en el marco del Sistema de Calidad Turístico Español y están vinculadas a una Denominación de Origen (D. O.) ${ }^{2}$ por lo que están estrechamente relacionadas con dos conceptos: calidad y certificación (TALLARICO, 2000). En este sentido, los gestores de las zonas vitivinícolas que quieran articular una oferta turística adherida a una Ruta del Vino deben cumplir con los criterios y estándares de calidad establecidos en el Manual de Producto Turístico Rutas del Vino de España (ACEVIN, 2012a, p. 20), en el que también se indica la metodología para su implantación y posterior certificación por parte del Comité de Gestión y Certificación de las Rutas del Vino de España ACEVIN (2009b, p.112).

Por otro lado, el sistema de certificación establecido dentro de la ruta regula el cumplimiento de los requisitos que se establecen tanto para que una ruta del vino sea considerada como tal como para que un establecimiento sea adherido a dicha ruta (FORONDA Y LÓPEZ, 2009). Este sistema se articula mediante de los siguientes documentos: Reglamento de Marca de Rutas del Vino de España, solicitud y criterios de certificación de las Rutas del Vino de España, procedimiento de auditoría de

\footnotetext{
${ }^{2}$ Aquella región, comarca o lugar reconocido administrativamente para designar a vinos que cumplan los siguientes requisitos: producir vinos a partir de materias primas de las zonas registradas, disfrutar de prestigio comercial, contar con calidad y características exclusivas al medio geográfico y haber transcurrido 5 años desde su reconocimiento como vino de calidad con indicación geográfica (TALLARICO, 2000, p. 13-14).
} 
certificación y de su renovación de las Rutas del Vino de España, solicitud de renovación, procedimiento de calificación de auditores, Reglamento de funcionamiento del comité de certificación, normas del comité de gestión de las Rutas del Vino de España (ACEVIN, 2009b).

Finalmente, una ruta debe ser capaz de actuar y controlar cinco ámbitos de actuación del sistema de gestión de la calidad del producto turístico: planificación y gestión, destino, promoción y comercialización, servicios turísticos y enología (ACEVIN, 2009b, p. 26), estos cinco subsistemas recogen los estándares de calidad del producto Ruta del Vino.

\subsection{RUTA DEL VINO RIBEIRA SACRA}

La Ruta del vino Ribeira Sacra fue creada en el año 2008 y su Ente Gestor está formado por el Consello Regulador de la Denominación de Origen Ribeira Sacra, la Asociación de Bodegueros de la Ribeira Sacra, el Consorcio de Turismo Ribeira Sacra, la Diputación de Lugo (INLUDES), la Diputación de Ourense (INORDE) y LugoSur y abarca alrededor de veinte municipios de las provincias gallegas de Lugo y Ourense. En la imagen 1 se muestra el mapa político de España y en la 2 la ruta del vino Ribeira Sacra norte y sur (TURGALICIA, 2010).

\section{IMAGEN 1 - MAPA POLITICO DE ESPAÑA}

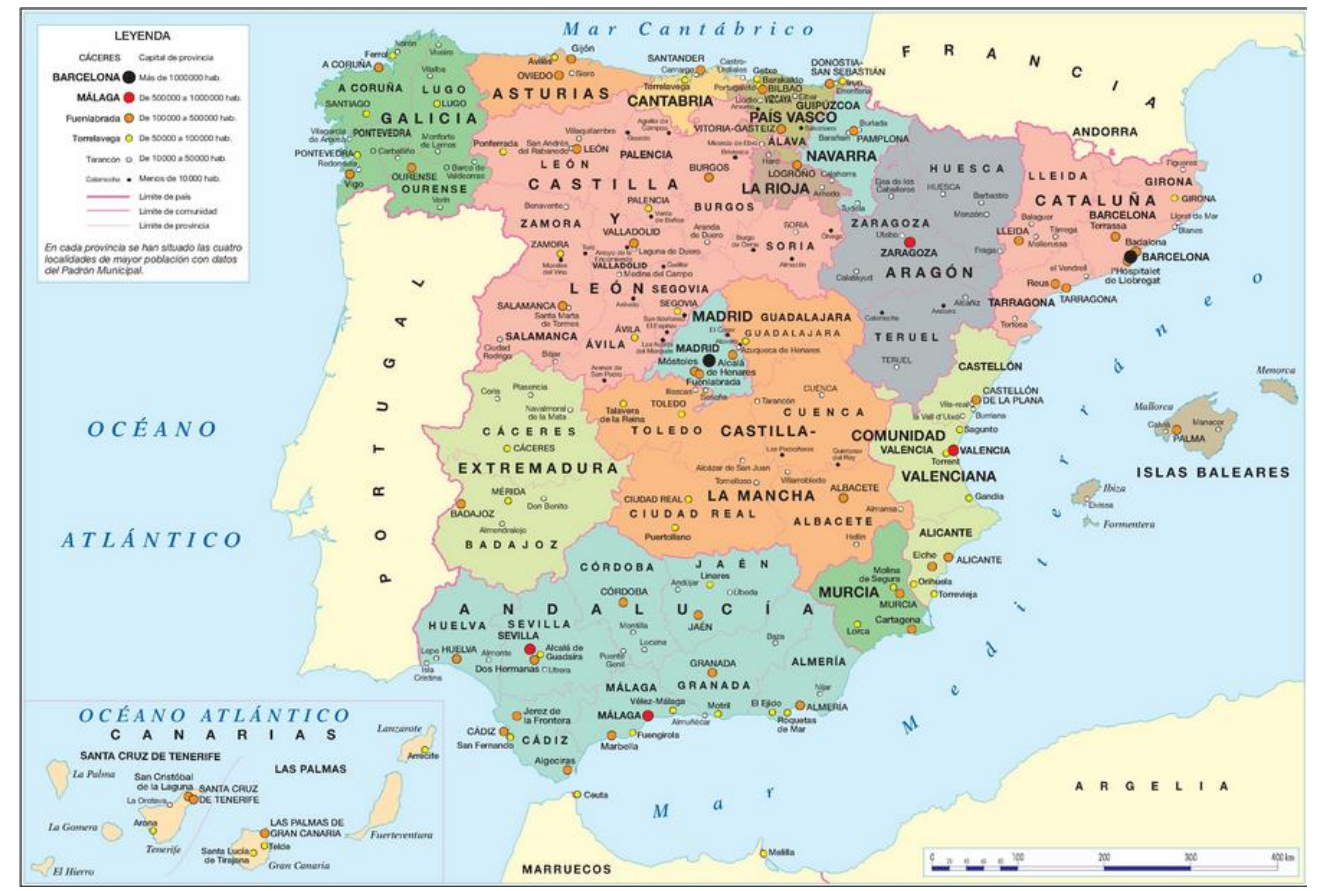

Fuente: http://luisamariaarias.wordpress.com/2012/11/27/ . Accedido en 20/10/2014. 
IMAGEN 2 - RUTA DEL VINO RIBEIRA SACRA RUTA NORTE

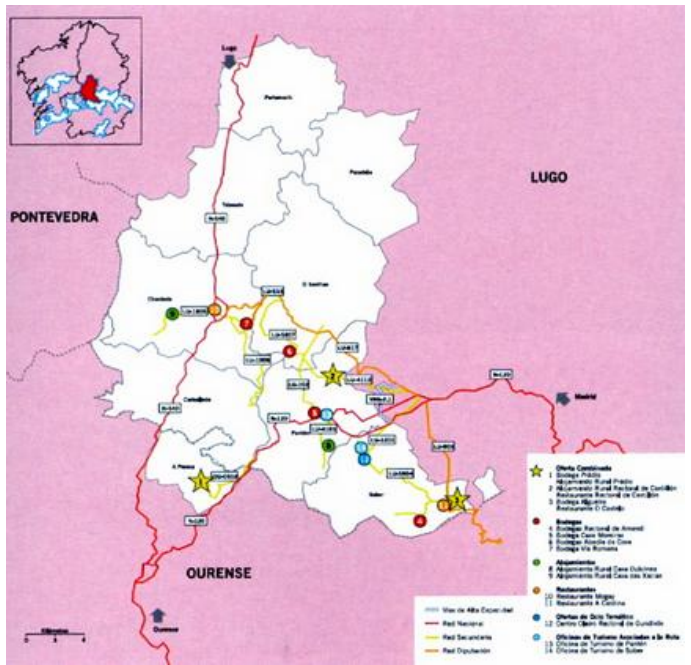

\section{RUTA SUR}

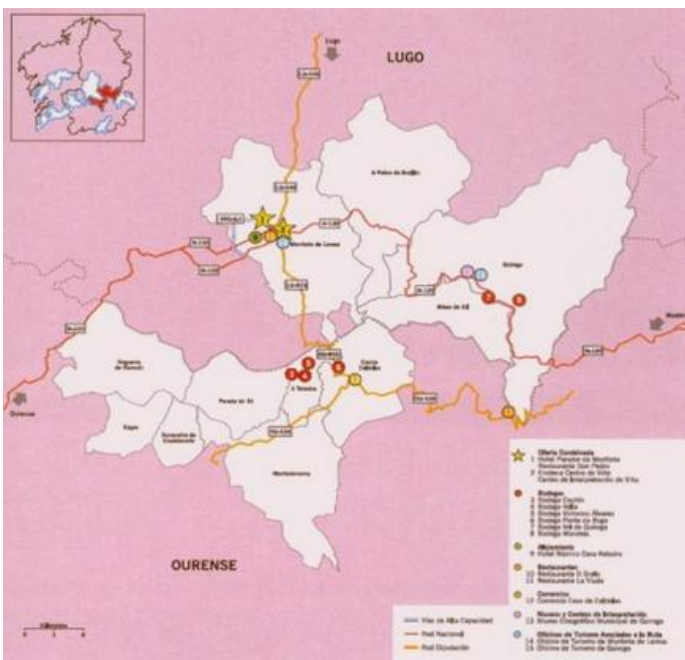

Fuente: Turgalicia (2010, p. 91-92): Guía de Rutas de los Vinos de Galicia.

Esta ruta está vinculada con la Denominación de Origen Ribeira Sacra que abarca una zona de producción de aproximadamente 1.263 hectáreas de viñedo (Tabla 1) producidas en las laderas de los ríos Miño y Sil y comprende 20 municipios de las zonas del sur de la provincia de Lugo y norte de Ourense. Dichos municipios se agrupan en 5 subzonas (BOE NÚM. 48, 2010): (1) Amandi que abarca los municipios de Sober y Monforte de Lemos, (2) Chantada que abarca ciertos municipios de Portomarin, Taboada, Carballedo, Chantada y A Peroxa, (3) Quiroga-Bibei que Discurre por los municipios de Monforte de Lemos, A Pobra de Brollón, Quiroga, Ribas de sil y Manzaneda, (4) Ribeiras do Miño que abarca los municipios de Paradela, O Saviñao, Pantón, Sober y Monforte de Lemos, (5) Ribeiras do Sil, la integran los municipios de A Teixeira, Parada de Sil, Castro Caldelas y Nogueira de Ramuín.

TABLA 1 - CIFRAS DEL CONSEJO REGULADOR

\begin{tabular}{lllll}
\hline & $\mathbf{2 0 1 0}$ & $\mathbf{2 0 1 1}$ & $\mathbf{2 0 1 2}$ & $\mathbf{2 0 1 3}$ \\
\hline Bodegas & 89 & 94 & 96 & 90 \\
Extensión (has) & 1.270 & 1.265 & 1.263 & 1.250 \\
Producción (Miles de kg) & 5.624 & 6.999 & 5.542 & 4.735 \\
Viticultores & 2.840 & 2.890 & 2.964 & 2.817 \\
Litros de vino embotellado & 3.184 .567 & 3.486 .487 & 5.139 .029 & 3.103 .268 \\
\hline
\end{tabular}

Fuente: Página web oficial de la D. O. Ribeira Sacra (www.ribeirasacra.org). Accedido en 21/10/2014.

En cuanto a las variedades de uva de la D. O. Ribeira Sacra, destacan blancas y tintas agrupadas en variedades preferentes y autorizadas. Las variedades de uva 
preferentes blancas son Godello, Albariño, Treixadura, Loureira, Dona Branca, Torrontés; y las variedades de uva preferente tintas son Mencía, Brancellao, Merenzao, Sousón, Caiño tinto, Tempranillo. En cuanto a las variedades de uva autorizadas destacan las tintas Garnacha Tintorera y Mouratón (BOE NÚM. 48, 2010).

\section{METODOLOGÍA DE INVESTIGACIÓN}

La población objetivo fueran los establecimientos (bodegas, restaurantes y alojamientos) adheridos a la Ruta del Vino Ribeira Sacra. La base de datos se elaboró a partir de la página web de la Ruta del Vino Ribeira Sacra $^{3}$ quedando constituida por 41 establecimientos adheridos a la ruta (13 bodegas, 12 restaurantes y 16 alojamientos); este dato se ve reducido una vez que se confirma con las empresas el mismo, quedando finalmente 12 bodegas, 10 restaurantes y 12 alojamientos en la base de datos (tabla 2).

Se envió un cuestionario estructurado a cada uno de los establecimientos. El proceso de recogida comenzó el 13 de Marzo y finalizo el 1 de Mayo de 2013, obteniendo 27 cuestionarios validos lo que representa un índice de respuesta del $77,14 \%$.

\section{TABLA 2 - FICHA TÉCNICA DEL ESTUDIO REALIZADO}

\begin{tabular}{|c|c|c|c|c|}
\hline Ribeira Sacra & BODEGAS & RESTAURANTES & ALOJAMIENTOS & TOTAL \\
\hline Universo de población & \multicolumn{4}{|c|}{$\begin{array}{c}\text { Bodegas, restaurantes y alojamientos adheridos a la Ruta del Vino Ribeira } \\
\text { Sacra (www.rutadelvinoribeirasacra.org) }\end{array}$} \\
\hline Ámbito geográfico & \multicolumn{4}{|c|}{$\begin{array}{c}\text { Denominación de Origen Ribeira Sacra (provincias de Lugo y Ourense, } \\
\text { Galicia) }\end{array}$} \\
\hline $\begin{array}{l}\text { Tamaño población al } \\
\text { inicio del estudio }\end{array}$ & 13 & 12 & 16 & 41 \\
\hline $\begin{array}{l}\text { Tamaño poblacional de } \\
\text { estudio }^{5}\end{array}$ & 13 & 10 & 12 & 35 \\
\hline Tamaño muestral & 12 & 8 & 7 & 27 \\
\hline Índice de respuesta (\%) & $92,30 \%$ & $80 \%$ & $58,33 \%$ & $77,14 \%$ \\
\hline Error muestral $(\alpha 0,05)$ & 8,01 & 15,81 & 24,40 & 9,20 \\
\hline Nivel de confianza & \multicolumn{4}{|c|}{$95 \% \mathrm{Z}=1,96 \mathrm{p}=\mathrm{q}=0,5$} \\
\hline
\end{tabular}

\footnotetext{
${ }^{3}$ http://rutadelvinoribeirasacra.org. Accedido en 15/04/2014.

${ }^{4}$ Hace referencia al tamaño de la población en base a la página web de la ruta del vino Ribeira Sacra (www.rutadelvinoribeirasacra.org).

${ }^{5}$ Se refiere a las altas y bajas de empresas en la ruta, confirmando este dato a través de la recogida de cuestionarios (e-mail, teléfono, contacto personal, etc.).
} 


\begin{tabular}{cll} 
Método de recogida & E-mail, teléfono, entrevista personal en establecimiento o puesto en feria del \\
Base de datos & Página web de la Ruta del vino Ribeira & Sacra \\
& (www.rutadelvinoribeirasacra.org) \\
Fecha de recogida & 13 de Marzo al 1 de Mayo de 2013 \\
\hline
\end{tabular}

Fuente: investigación de campo.

En lo que respecta al diseño del cuestionario, se ha tenido en cuenta la Guía de Sostenibilidad para empresas del sector enoturístico (ACEVIN, 2010, p. 31-295), el Estudio de la Oferta Enoturística de las Rutas del Vino de España (ACEVIN, 2009a, p. 19-29) y el Estudio de la Oferta Enoturística de las Rutas del Vino de España. 2 ${ }^{\circ}$ semestre (ACEVIN, 2009c, p. 4-44). Se elaboraron tres cuestionarios diferentes para cada uno de los establecimientos encuestados.

Los cuestionarios tenían cinco partes, todas ellas relacionadas. Una primera parte haciendo referencia al perfil general de la empresa, la segunda parte dirigida a conocer en profundidad todo lo relacionado con las actividades de enoturismo, la tercera parte buscando obtener el perfil de los visitantes, en la penúltima se ha solicitado la información necesaria para la elaboración de un análisis FODA (Fortalezas, Oportunidades, Debilidades, Amenazas) a través de las opiniones de los responsables de los establecimiento y por último se solicita una valoración global de la situación de la actividad enoturística en la Ruta del Vino Ribeira Sacra.

\section{ANÁLISIS DE DATOS}

En este apartado se presentan y analizan los siguientes datos: Perfil de los establecimientos adheridos a la Ruta del Ribeira Sacra; Oferta de actividades enoturísticas de las bodegas, restaurantes y alojamientos; Perfil de los enoturistas desde el punto de vista de los establecimientos objeto de estudio; Nivel de satisfacción de los establecimientos tras su adhesión a la Ruta; Valoración de la actividad enoturística de la Ruta y se elaboró un análisis FODA a partir de la información proporcionada por los establecimientos encuestados. 


\subsection{PERFIL DE LOS ESTABLECIMIENTOS ADHERIDOS A LA RUTA DEL RIBEIRA SACRA}

Para el análisis del perfil se ha tenido en cuenta las características que definen el tipo de establecimiento como son el tipo de propiedad y sociedad, número de empleados y formación (Tabla 3). Según los datos, la empresa familiar ${ }^{6}$ es mayoritaria en los tres tipos de establecimientos y la forma jurídica predominante es la Sociedad Limitada (S.L.) excepto en las bodegas, que presentan el mismo porcentaje las empresas individuales y las S. L. (33,33\%). En lo que respecta al nivel formativo en el caso de los alojamientos éstos tienen un mayor número de empleados con estudios universitarios que las bodegas y restaurantes, que por contra disponen de un mayor número de empleados con un titulo profesionalizante (FP) en las bodegas y en el caso de los restaurantes se reparten por igual entre tres niveles (Diplomados y Licenciados, FP y estudios secundarios). Excepto los restaurantes que llevan a cabo cursos de formación de forma habitual las bodegas y alojamientos lo hacen de forma ocasional. Teniendo en cuenta el número de trabajadores todas las bodegas y restaurantes son micro PYMES (Pequeñas y Medianas Empresas) y en el caso de los alojamientos el 28,57\% son PYMES con más de 10 trabajadores.

TABLA 3 - PERFIL DE DE LOS ESTABLECIMIENTOS ADHERIDOS A LA RUTA DEL VINO RIBEIRO

\begin{tabular}{|c|c|c|c|c|}
\hline PERFIL & CONCEPTO & BODEGAS & RESTAURANTES & ALOJAMIENTOS \\
\hline \multirow{2}{*}{ Propiedad } & Familiar & $83,33 \%$ & $62,50 \%$ & $85,71 \%$ \\
\hline & No familiar & $16,67 \%$ & $37,50 \%$ & $14,29 \%$ \\
\hline \multirow{4}{*}{ Tipo sociedad } & Emp. Individual & $33,33 \%$ & $42,90 \%$ & $28,57 \%$ \\
\hline & S. L. & $33,33 \%$ & $57,10 \%$ & $42,86 \%$ \\
\hline & S. A. & $16,67 \%$ & & $14,29 \%$ \\
\hline & Otra & $16,67 \%$ & & $14,29 \%$ \\
\hline \multirow[b]{2}{*}{$\begin{array}{l}\text { Frecuencia de } \\
\text { formación }\end{array}$} & Continuamente & $25 \%$ & $75,00 \%$ & $28,57 \%$ \\
\hline & $\begin{array}{l}\text { Ocasionalmente } \\
\text { Nunca }\end{array}$ & $\begin{array}{c}66,67 \% \\
8,33 \%\end{array}$ & $25,00 \%$ & $71,43 \%$ \\
\hline Nivel formativo & $\begin{array}{l}\text { Diplomados y } \\
\text { licenciados }\end{array}$ & $7,69 \%$ & $27,27 \%$ & $36,36 \%$ \\
\hline
\end{tabular}

\footnotetext{
${ }^{6}$ Empresa Familiar: Se pueden considerar empresas familiares todas las sociedades y empresas individuales que desarrollen sus actividades económicas, industriales y de producción de bienes y servicios, o administren y gestionen participaciones directas o indirectas de sociedades que desarrollan tales actividades y que están participadas de manera significativa por un grupo familiar que actúa con vocación de continuidad bajo una unidad de decisión y dirección en las que al menos un miembro del grupo familiar interviene de manera efectiva en la gestión de la empresa ostentando la capacidad de decisión sobre los aspectos más relevantes que le conciernen a la sociedad familiar. (GALLEGO, 2012, p. 6-7).
} 


\begin{tabular}{c|l|c|c|c}
\hline & Formación & $38,46 \%$ & $27,27 \%$ & $9,09 \%$ \\
& Profesional (FP) & $30,77 \%$ & $27,27 \%$ & $45,45 \%$ \\
& Secundarios & $23,8 \%$ & $18,18 \%$ & $9.09 \%$ \\
\hline & Primarios & $92 \%$ & $100 \%$ & $71,43 \%$ \\
\hline Número de & $<10$ & 8 & & $28,57 \%$ \\
empleados & $\geq 10$ & &
\end{tabular}

Fuente: Elaboración propia.

Para completar el perfil general de los establecimientos se analiza también las características específicas dichos establecimientos. En lo que respecta a las bodegas pertenecientes a la Ruta, tiene una superficie de instalación entre 0 y $500 \mathrm{~m}^{2}$ el $50 \%$, entre 501 y $2.000 \mathrm{~m}^{2}$ el 33,33\% y más de $2000 \mathrm{~m}^{2}$ el 16,67\%, y una superficie en hectáreas de viñedo de menos de 50 hectáreas en el 92\% de las bodegas. La producción anual en miles de litros es inferior a 100.000 litros en el 58,33\% de las bodegas, sólo el 8,33\% tiene una producción de más de 500.000 litros, producción controlada por un enólogo en todos las bodegas, que en 7 de las 12 está subcontratado. Las variedades de uva más cultivadas son el Godello como uva blanca (32\% del total de las variedades), el albariño con un $28 \%$ y treixadura con un $20 \%$, todas ellas variedades autoctonas de la zona.

Para concluir el análisis del perfil de las bodegas adheridas a la ruta del vino Ribeira Sacra se han identificado los canales que estaban sendo empleados para la distribución de su producción; el canal de los distribuidores es el más empleado por todas las bodegas, representa un $21,43 \%$, la tienda en la propia bodega es el segundo canal más empleado, concretamente por nueve de las bodegas encuestadas; le siguen la hostelería y vinotecas y los canales menos empelados eran los mayoristas, solo tres bodegas los empleaban, y las empresas de catering no son un canal de distribución empleado por ninguna bodega de las entrevistadas. En cuanto a si el porcentaje de producción que comercializan es a nivel nacional o internacional, diez responsables por las bodegas afirmaron que vendían casi toda su producción en el mercado nacional, entre el $80 \%$ y $100 \%$.

En lo referente al análisis de la capacidad de los restaurantes, éstos tenían disponibilidad en su local para más de cincuenta personas, en concreto cinco de ellos disponían de un local con capacidad de entre 50-100 personas, 1 hasta 150 personas y 2 restaurantes disponía de espacio para más de 150 personas, concretamente entre 150300 comensales. La ocupación media de los restaurantes en cuatro de ellos rondaba 
entre el $30 \%$ y $40 \%$ mientras que otros tres se situaba entre el $60 \%$ y $70 \%$ de ocupación media anual.

En los alojamientos la ocupación media anual se puede calificar como baja en general, ya que ningún establecimiento superaba el $60 \%$ anualmente según los datos recogidos y el precio medio de una habitación doble ronda entre los cuarenta y ochenta euros. En general, no solo ofrecían servicio de alojamiento, también ofrecían servicio de restauración y servicios vinculados con el enoturismo.

\subsection{OFERTA DE ACTIVIDADES ENOTURÍSTICAS DE LAS BODEGAS, RESTAURANTES Y ALOJAMIENTOS}

En este epígrafe, se aborda el análisis de las actividades relacionadas con el enoturismo que los establecimientos ofrecían a sus clientes, como captaban clientes y lograban su fidelización, que sistemas de publicidad utilizaban, y el gasto medio de los clientes en cada visita (cuadro 1).

\section{CUADRO 1 - OFERTA ENOTURÍSTICA.}

\begin{tabular}{|c|c|c|c|c|}
\hline \multicolumn{2}{|c|}{ ACTIVIDADES } & $\begin{array}{c}\text { BODEGAS } \\
\text { (MUESTRA 12) }\end{array}$ & $\begin{array}{l}\text { RESTAURANTES } \\
\text { (MUESTRA 8) }\end{array}$ & $\begin{array}{c}\text { ALOJAMIENTOS } \\
\text { (MUESTRA 7) }\end{array}$ \\
\hline \multicolumn{2}{|c|}{ Realizaron alguna inversión } & $\mathrm{Si} 75 \%$ & Si $100 \%$ & Si $50 \%$ \\
\hline \multicolumn{2}{|c|}{$\begin{array}{l}\text { La adhesión ha generado } \\
\text { incremento de la rentabilidad }\end{array}$} & $\mathrm{Si} 45,45 \%$ & Si $37,50 \%$ & No $100 \%$ \\
\hline \multicolumn{2}{|c|}{$\begin{array}{l}\text { Ha notado algún beneficio tras } \\
\text { la adhesión }\end{array}$} & Si $54,55 \%$ & Si $62,50 \%$ & No $100 \%$ \\
\hline \multicolumn{2}{|c|}{$\begin{array}{l}\text { Incremento de los clientes tras } \\
\text { la adhesión }\end{array}$} & $\begin{array}{l}\text { Reciben más } \\
\text { clientes }\end{array}$ & $\begin{array}{c}\text { Reciben más } \\
\text { clientes y gastan } \\
\text { más }\end{array}$ & $\begin{array}{l}\text { Reciben los mismos } \\
\text { clientes }\end{array}$ \\
\hline \multicolumn{2}{|c|}{$\begin{array}{l}\text { Ha generado empleo en su } \\
\text { empresa la adhesión }\end{array}$} & \multicolumn{3}{|c|}{ Ninguna contratación } \\
\hline \multicolumn{2}{|c|}{$\begin{array}{l}\text { Servicios de enoturismo } \\
\text { ofrecidos }\end{array}$} & $\begin{array}{l}\text { Las } 12 \text { bodegas } \\
\text { que forman parte } \\
\text { de la ruta ofertan: } \\
\text { Venta de vinos } \\
\text { Visita a bodegas } \\
\text { Degustación de } \\
\text { vinos }\end{array}$ & $\begin{array}{l}\text { Menos marinados } \\
\text { (6) } \\
\text { Restaurante } \\
\text { integrado en } \\
\text { paquetes } \\
\text { enoturísticos (5) } \\
\text { Degustación de } \\
\text { vinos (4) } \\
\text { Venta de vinos (4) }\end{array}$ & $\begin{array}{l}\text { Venta de vinos (6) } \\
\text { Restaurante o jornadas } \\
\text { gastronómicas (4) } \\
\text { Organizacion de eventos } \\
\text { vinícolas (3) } \\
\text { Alojamiento integrado en } \\
\text { paquetes enoturísticos (3) }\end{array}$ \\
\hline \multirow{5}{*}{$\begin{array}{l}\text { Captación } \\
\text { clientes }\end{array}$} & Páginas web & $25,93 \%$ & $35 \%$ & $28 \%$ \\
\hline & $\begin{array}{l}\text { Oficinas de } \\
\text { turismo }\end{array}$ & $14,81 \%$ & $20 \%$ & $20 \%$ \\
\hline & Agencias de viajes & $18,52 \%$ & $10 \%$ & $20 \%$ \\
\hline & Folletos & $22,22 \%$ & $20 \%$ & $4 \%$ \\
\hline & Prensa y/o radio & $18,52 \%$ & $15 \%$ & $8 \%$ \\
\hline
\end{tabular}




\begin{tabular}{|l|l|l|l|}
\hline Actividades de fidelización & No realizan & $\begin{array}{c}\text { Jornadas } \\
\text { gastronómicas }\end{array}$ & No realizan \\
\hline
\end{tabular}

Fuente: Elaboración propia.

Todos los establecimientos han necesitado realizar pequeñas inversiones tras su adhesión a la ruta para satisfacer a los turistas y más del 50\% de los entrevistados en las bodegas y restaurantes y el 100\% de los alojamientos han identificado que la adhesión no ha generado un incremento en su rentabilidad, pero sí han notado un incremento en el número de clientes que recibían.

El medio para la captación de clientes más empleado en todos los establecimientos era la internet a través de publicidad en páginas web, ya sea la propia página del establecimiento u otras relacionadas con el sector. En general no realizaban actividades de fidelización a excepción de los restaurantes que si realizaban jornadas gastronómicas.

\subsection{PERFIL DE LOS ENOTURISTAS DESDE EL PUNTO DE VISTA DE LOS ESTABLECIMIENTOS OBJETO DE ESTUDIO}

La procedencia de los turistas era nacional, principalmente de Galicia y los escasos extranjeros que visitaban la zona procedían de Alemania, Francia e Inglaterra, siendo la principal época de afluencia el verano (julio y agosto). La imagen que ofrecía la Ribeira Sacra para los establecimientos es de reducida contaminación, gran contacto con la naturaleza y zona muy tranquila.

CUADRO 2 - PERFIL DEL ENOTURÍSTA

\begin{tabular}{|c|c|c|c|}
\hline PERFIL & BODEGAS & RESTAURANTES & ALOJAMIENTOS \\
\hline Procedencia principal del visitante & \multicolumn{3}{|c|}{ Nacional } \\
\hline $\begin{array}{l}\text { Procedencia principal visitante por CCAA } \\
\text { (Comunidad Autonoma) }\end{array}$ & \multicolumn{3}{|c|}{ Galicia } \\
\hline $\begin{array}{l}\text { Procedencia principal del visitante } \\
\text { internacional }\end{array}$ & \multicolumn{3}{|c|}{$\begin{array}{l}\text { Alemania } \\
\text { Francia } \\
\text { Inglaterra }\end{array}$} \\
\hline Principal época de afluencia & \multicolumn{2}{|c|}{ Verano y Semana Santa } & $\begin{array}{c}\text { Verano y puentes } \\
\text { y festivos }\end{array}$ \\
\hline Imagen de la ruta para los establecimientos & \multicolumn{3}{|c|}{$\begin{array}{c}\text { Naturaleza } \\
\text { Reducida contaminación } \\
\text { Zona tranquila }\end{array}$} \\
\hline
\end{tabular}

Fuente: Elaboración propia. 


\subsection{NIVEL DE SATISFACCIÓN DE LOS ESTABLECIMIENTOS TRAS SU ADHESIÓN A LA RUTA}

Lo más destacable de este punto, es el alto porcentaje de insatisfacción por parte de los alojamientos que pertenecían a la Ruta del Ribeira Sacra. En cuanto a la satisfacción de las bodegas se pudo concluir que los responsables por cinco de ellas estaban satisfechas tras la adhesión, mientras que cuatro afirmaron tener opinión neutral y en el caso de los restaurantes, cuatro estaban satisfechos tras la adhesión, mientras que tres han dado una opinión neutra y uno se encontraba muy poco satisfecho.

\section{GRÁFICOS 1 - NIVEL DE SATISFACCIÓN}

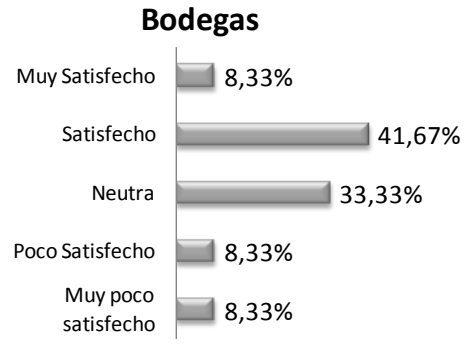

Alojamientos

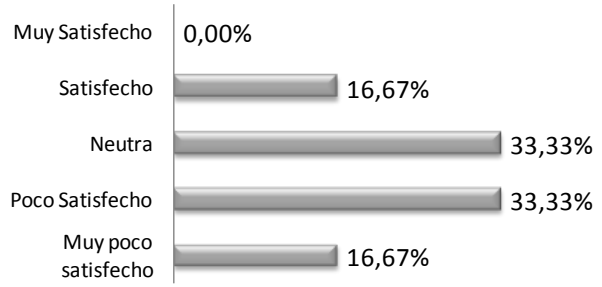

Fuente: Elaboración propia.

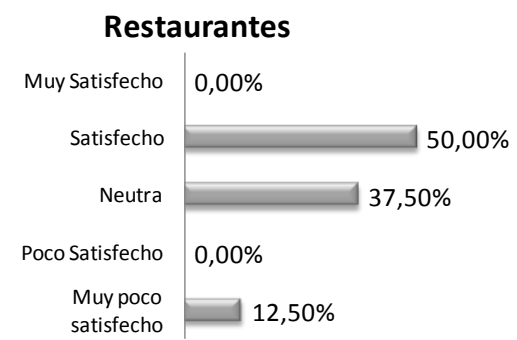

Establecimientos de la R.V. Ribeira Sacra

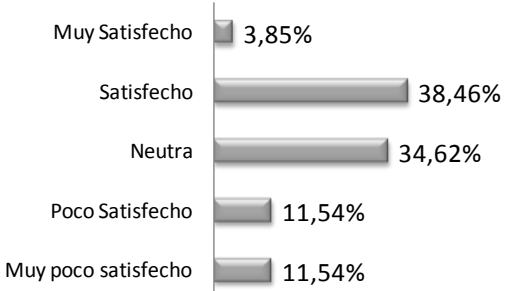

\subsection{VALORACIÓN DE LA ACTIVIDAD ENOTURÍSTICA DE LA RUTA}

En relación a la valoración global de la actividad enoturística, teniendo en cuenta una escala Likert de 5 puntos que va desde 1 (muy baja) a 5 (muy buena), la percepción de los establecimientos ha diferido de unos a otros. Así, el 18,52\% la consideraba buena o muy buena, el 51,85\% no la consideraban ni buena ni baja, sin embargo el 29,62\% la consideraba baja o muy baja. En el caso de las bodegas, cinco se quedaban en el medio ni la consideraban baja ni buena y cuatro de ellas tenían una buena valoración acerca de la misma, solo tres mostraban una valoración baja (2) o muy baja (1). Para 6 
restaurantes la valoración global era neutra, mientras que para dos de los restaurantes era buena. Se observó que los alojamientos eran los que han evaluado más negativamente la actividad enoturística de la ruta, tres de ellos ni la consideraban buena ni baja y 4 la consideraban baja o muy baja.

\subsection{FODA A PARTIR DE LA INFORMACIÓN PROPORCIONADA POR LOS ESTABLECIMIENTOS ENCUESTADOS}

Se elaboraron tres FODAs a partir de las aportaciones de cada uno de los miembros de la ruta: bodegas, restaurantes, alojamientos y en el Cuadro 3 aparecen recogidas todas las opiniones de los miembros de la Ruta del Vino en un único FODA.

\section{CUADRO 3 - ANÁLISIS FODA DE LA RUTA DEL VINO RIBEIRA SACRA}

\begin{tabular}{|c|c|}
\hline \multicolumn{2}{|c|}{ BODEGAS, RESTAURANTES Y ALOJAMIENTOS } \\
\hline DEBILIDADES & FORTALEZAS \\
\hline $\begin{array}{l}\text { Deficiente y confusa señalización de las carreteras } \\
\text { y accesos. } \\
\text { Escasa oferta gastronómica y de alojamiento. } \\
\text { Falta de promoción y coordinación en general } \\
\text { (alojamientos, bodegas y restaurantes) } \\
\text { Falta de actividades en común } \\
\text { Formación turística } \\
\text { Falta de oficinas de turismo abiertas } \\
\text { Dificultad de encontrar catálogos los turistas } \\
\text { Imagen de un tipo de vino tinto joven Mencía } \\
\text { Falta de unificación en folletos promocionales } \\
\text { Falta de empresas de coordinación de ocio } \\
\text { Falta de calendario de actividades } \\
\text { Falta de infraestructuras turísticas. } \\
\text { Comunicación } \\
\text { Mas colaboración con pequeñas bodegas } \\
\text { Baja profesionalidad } \\
\text { Poca cooperación entre los agentes participantes, } \\
\text { sobre todo bodegas y restauración } \\
\text { Falta más apoyo institucional } \\
\text { Poca proyección fuera del mercado local } \\
\text { Necesidad de modernizar las cartas de menús } \\
\text { Necesidad por acondicionar mejor el local } \\
\text { Pocos medios de transporte } \\
\text { Faltan rutas organizadas } \\
\text { Difícil acceso a información } \\
\text { Escasa profesionalidad en los servicios que se } \\
\text { ofertan } \\
\text { Bastante estacionalidad } \\
\text { Entorno poco cuidado } \\
\text { Poca oferta alternativa } \\
\text { Pensar que todo lo tiene que hacer la }\end{array}$ & $\begin{array}{l}\text { Naturaleza magnífica, gran atractivo turístico- } \\
\text { cultural-paisajístico de la zona } \\
\text { Vinos con distintas personalidades y de calidad } \\
\text { Carácter amigable de las gentes. } \\
\text { Entorno geográfico excepcional } \\
\text { Elaboración de vinos de alta calidad } \\
\text { Consolidada trayectoria } \\
\text { Gastronomía y su vinculación al enoturismo } \\
\text { Oferta turística } \\
\text { Románico y sus rutas } \\
\text { Variedades y razas autóctonas } \\
\text { Gran atractivo turístico-cultural-paisajístico, de la } \\
\text { zona. } \\
\text { Gran potencial del enoturismo } \\
\text { Vinculación del enoturismo con la gastronomía. } \\
\text { Viticultura heroica } \\
\text { Calidad de atención a visitantes y de los servicios } \\
\text { marca conjunta } \\
\text { Posicionamiento de la D. O. } \\
\text { Historia del vino y producto diferenciador respecto } \\
\text { de otras zonas vinícolas } \\
\text { Catamarán y paisajes } \\
\text { Uvas autóctonas } \\
\text { Viticultura de alta montaña, binomio naturaleza- } \\
\text { vino } \\
\text { Elaboración de productos agro artesanales y } \\
\text { agroalimentarios } \\
\text { Antigüedad en el sector } \\
\text { Respeto hacia el cliente } \\
\text { Muchas casas rurales } \\
\text { Abundante y excelente oferta gastronómica } \\
\text { Rutas del vino }\end{array}$ \\
\hline
\end{tabular}




\begin{tabular}{l}
\hline administración \\
Falta de mentalidad de cuidar la naturaleza \\
Conocimiento fuera de la Comarca (Ribeira Sacra) \\
No se da valor a lo que se tiene \\
No se sabe trabajar en equipo \\
Falta de profesionales \\
Falta de seriedad en los servicios ofrecidos \\
Un vino muy costoso y por lo tanto caro \\
Falta de conocimiento del mercado del producto \\
Falta de unificación en folletos promocionales \\
Falta de acuerdos comunes entre las dos \\
diputaciones que forman la Ribeira Sacra \\
Órgano competente para el desarrollo y promoción \\
de la Ribeira Sacra \\
Zona demasiado amplia
\end{tabular}

\begin{tabular}{|l|l|}
\hline AMENAZAS & \\
\hline Despoblación preocupante &
\end{tabular}

Escaso interés inversor de las autoridades

Deterioro de las infraestructuras

Resto de rutas más profesionalizadas

Cansancio del turista con bodegas cerradas

Embalses, aerogeneradores

Gasto público sin planificación

Minifundismo (también mental)

Abandono rural

Educación

Dispersión en la información.

Desinterés de las empresas

No ofrecer una mejor calidad.

Gran diversidad de rutas enológicas presentes en la comunidad

Al ser una zona poco señalizada, posibilidad de perderse en la misma

Coyuntura económica actual

Localismos

Otras D. O. más consolidadas (antiguas)

Coyuntura económica

Turismo estacional

Intrusismo laboral

Fuerte competencia

Malas previsiones meteorológicas

Vulnerabilidad ante grandes competidores

Situación de crisis actual

Baja participación de las administraciones

Promoción de otras actividades como golf y

balneario en detrimento de nuestra oferta

Falta de recursos económicos de las instituciones

públicas

Cliente exigente

Otras rutas del vino mejor desarrolladas

El actual destino turístico asociado a Galicia, está enfocado a sol y playa

Monopolización por determinados alojamientos y bodegas

Visitas a bodegas como si fuesen monasterios

Visitantes defraudados
Situación geográfica con respecto a otros destinos (Ourense, Santiago...)

Historia

Pertenecer a la D.O. Ribeira Sacra

Mayor concentración de monasterios e iglesias románicas de España

Dos de los 93 paradores de turismo de España se encuentran ubicados en esta zona

Incremento año a año de visitantes

Oportunidad para productos ecológicos alternativos El turista de naturaleza, no sólo el enoturista

Nuevas tecnologías

Incremento de opciones lúdicas con una vertiente turístico - enológica

Mercado internacional

E-comerce y redes sociales

Jóvenes cualificados e inquietos

Potenciar más la oferta enoturística

Profesionalización del sector

Compromiso y seriedad

Conocimiento de la zona

Divulgación de los productos

Encave único

Cercanía a Madrid, AVE próximo

Existencia de dos paradores en la zona

Diversidad cultural

Viticultura

Turismo natural

Potenciar más la oferta de enoturismo gastronómico.

Profesionalizar el sector

Compromiso y seriedad

Mejora en comunicaciones para la percepción de visitantes

Destino turístico que cada vez recibe un mayor reconocimiento

\section{Turismo enológico en auge}

Desplazamientos cortos de los vecinos lo que conlleva aumento de beneficios para nosotros

Aprovechamiento de internet como vía de comunicación

Se podrían desarrollar nuevos productos

Los márgenes de ganancia son buenos

Aprovechar la emergente cultura vinícola

Aumentar la pre-venta

Situación económica que hace que la gente se quede en la zona más cercana

Necesidad de colaborar entre todos y aunar esfuerzos 


\begin{tabular}{|l|l|}
\hline & Mercado tanto nacional como internacional \\
Zona aun por explotar \\
Turismo \\
& Destino turístico en potencia, que una vez conocido \\
se hace muy atractivo & Alternativa al turismo de sol y playa \\
& Participación en las actividades de la bodega \\
Oferta por parte de las bodegas de visitas y catas
\end{tabular}

Fuente: Elaboración propia a partir de las respuestas de los entrevistados.

\section{CONCLUSIONES}

Entre la Comunidad Autónoma Gallega y Portugal nació un proyecto vinícola denominado "Rutas do Viño da Eurorrexión Galicia-Norte de Portugal" formada por las cinco rutas del vino gallegas y la ruta del vino portuguesa denominada Ruta dos Vinhos Verdes. Formando parte de las rutas gallegas, la Ruta del Vino Ribeira Sacra de reciente creación y en fase de certificación como Ruta del Vino de España, evoluciona tratando de consolidarse como un nuevo producto turístico en la zona para dar a conocer su cultura, patrimonio, actividades, vino y generar riqueza y empleo en la zona rural donde está ubicada (XUNTA DE GALICIA, 2012).

En este contexto, es necesario averiguar si se está consolidando como producto turístico en la zona y sí se están cumpliendo los objetivos con los que fue creada, para lo que se ha tenido tenido en cuenta la percepción de los establecimientos que conforman la Ruta. Los resultados obtenidos han permitido definir:

(1) El perfil de los establecimientos. Cuyo análisis pone de relieve la necesidad de una mayor profesionalización de los empleados.

(2) La oferta de actividades enoturísticas. Los datos obtenidos muestran que la mayoría de los establecimiento percibían que la adhesión a la ruta no ha supuesto un incremento de la rentabilidad, pero si un incremento del número de clientes. Discrepancia cuya explicación probablemente se debe al momento de crisis económica que atraviesa España. Por otro lado, la adhesión no ha generado ningún puesto de trabajo en ninguno de los establecimientos entrevistados.

(3) El perfil del enoturista. Este análisis ha permitido detectar que la demanda era estacional (verano, Semana Santa y Festivos) y la procedencia de los turistas es 
nacional, principalmente de Galicia. Entre los turistas internacionales no se encontrarón aquellos del país vecino (Portugal), lo que permite afirmar que existe un mercado sin explotar.

La principal aportación de este estudio ha sido averiguar el nivel de satisfacción de los gestores de los establecimientos tras su adhesión a la ruta, la valoración de la actividad enoturística por parte de éstos y la obtención de un FODA elaborado a partir de los principales componentes desde el punto de vista de la oferta de la Ruta que nos ha permitido realizar un análisis en profundidad de la Ruta. Los resultados obtenidos ponen en evidencia el alto grado de insatisfacción de los alojamientos, mientras que los entrevistados de las bodegas y los restaurantes presentaron niveles más altos de satisfacción. En relación a la valoración global de la actividad enoturística el 18,52\% la consideraba buena o muy buena, el $51,85 \%$ no la consideraba ni buena ni baja, sin embargo el 29,62\% la consideraba baja o muy baja.

De los objetivos marcados por toda Ruta del Vino, se ha podido ver que no se han alcanzado varios de ellos: no se ha conseguido desestacionalizar la demanda, no se ha conseguido generar empleo en la zona, y la generación de riqueza todavía no se ha consolidado.

En lo que respecta al análisis FODA ha que se destacar que la Ruta del Vino Ribeira Sacra debe para consolidarse:

- Mantener sus fortalezas: gran atractivo turístico-cultural-paisajístico, buena situación geográfica, carácter amigable de las gentes de la zona, gran potencial del enoturismo, antigüedad en el sector del vino, tierras de gran tranquilidad y paz, gran biodiversidad paisajística y reducida contaminación, entre otras.

- Vencer las debilidades: falta de señalización e información de la ruta, falta de acuerdos entre las dos diputaciones de la Ribeira Sacra, escasa oferta gastronómica y de alojamiento, formación de los empleados, estacionalidad, (entre otras).

- Luchar contra las amenazas: despoblación de la zona, deterioro de infraestructuras, la variedad de rutas del vino en nuestra comunidad y otras D. O. más antiguas, el turismo de sol y playa, visitantes defraudados por no recibir lo que esperaban, escaso interés inversor de las autoridades. 
- Aprovechar las oportunidades: el mercado internacional, aprovechamiento de internet como vía de comunicación y la emergente cultura vinícola, turismo alternativo al de sol y playa, aprovechamiento de las redes sociales para interaccionar, entre otras.

\section{REFERENCIAS}

ACEVIN - ASOCIACIÓN ESPAÑOLA DE CIUDADES DEL VINO. Estudio de la Oferta Enoturística de las Rutas del Vino de España (2009). Disponible en: <http:// >. (2009a).

Accedido en: 29/10/2014.

Manual de Producto Rutas del Vino España. Disponible en: <http://www.rutadelvinoriberadelduero.es/castellano/consorcio-ruta-del-vino/sistemade-adhesion-al-consorcio.html>. (2009b). Accedido en: 29/10/2014.

Estudio de la oferta enoturística de las rutas del vino de España. Disponible en: <http://www.rutadelvinoriberadelduero.es/desc_adj_observatorio.php?id=4>. (2009c). Accedido en: 29/10/2014.

Guía de Sostenibilidad para empresas del sector enoturístico. Acciones de Adaptación y Formación. España. Disponible en: <http://www.enoturismoresponsable.es>. (2010). Accedido en: 29/10/2014.

Club de producto Rutas del Vino de España. Evaluación del Turismo en España. Oferta y Demanda de Enoturismo. Disponible en: <http://www.euskadi.net/contenidos/memoria/ponencias_turismoyvino/es_agripes/adju ntos/Ponencia_ACEVINP.pdf>.(2012a). Accedido en: 29/10/2014.

Guía de rutas del vino de España. España: Asociación Española de Ciudades del Vino. Disponible en: <http://www.wineroutesofspain.com/bd/archivos/archivo121.pdf>. (2012b). Accedido en: $28 / 10 / 2014$.

ALPÍZAR PADILHA, V. A.; MALDONADO AVALOS, M. M. Integración de la ruta del vino en Querétaro, un producto innovador. Revista Quivera. Revista De Estudios Urbanos, Regionales, Territoriales, Ambientales y Sociales, (2), 97-109. (2011).

ANDREU, R.; VERDÚ, L. Turismo enológico en Alicante: la ruta del vino en el municipio de Pinoso. Cuadernos de Turismo, 35-61. Julio-Diciembre (2012).

BOE NÚM. 48 - BOLETÍN OFICIAL DEL ESTADO. Sec. III. Pág. 18102. Resolución de 2 de diciembre de 2009, de la Dirección General de Industria y Mercados Alimentarios, por la que se aprueba el Reglamento de la Denominación de Origen "Ribeira Sacra" y de su Consejo Regulador. Disponible en: 
$<$ http://ribeirasacra.org/media/documentos/Reglamentode\%20laRibeiraSacraCR2010.pd f>. Accedido en: 28/10/2014.

BRUWER, J. South African wine routes: some perspectives on the wine tourism industry's structural dimensions and wine tourism product. Tourism Management, 24 (3), 423-435. (2003).

DEL RÍO, M. C.; ÁlVAREZ, J.; FRAIZ, J. A. Ruta Turística Enológica Rías Baixas (Galicia-España): Análisis desde la perspectiva de la oferta. CULTUR- Revista de Cultura y Turismo, ano 8, (01), Fev., 95-118. (2014).

DEL RÍO, M. C.; ÁLVAREZ, J.; VILA, M.; IGLESIAS, L. Turismo Enológico y Ruta del Vino del Bierzo. REDMARKA - Revista Digital de Marketing Aplicado, año VI, (11), 185-212. (2013).

FORONDA, C.; LÓPEZ, A. M. La apuesta por la calidad como elemento diferenciador en los destinos turísticos: Planes renovados. Cuadernos de Turismo, 23, p. 102-153. (2009).

GALLEGO, I. La empresa familiar y delimitación jurídica. Cuadernos de Reflexión de la Cátedra PRASA de Empresa Familiar, (14), 1-45. (2012).

HALL, C. M.; MITCHELL, R. Wine Tourism in the Mediterranean: A Tool for Reestructuring and Development. Thunderbird International Business Review, 42 (4), 445-465. (2001).

LÓPEZ-GUZMÁN, T.; SÁNCHEZ CAÑIZARES, S. M. La creación de productos turísticos utilizando rutas enológicas. Pasos. Revista de Turismo y Patrimonio Cultural, 6(2), 159-171. (2008).

MEDINA F. X.; TRESSERRAS J. Turismo enológico y rutas del vino en Cataluña. Análisis de casos: D. O. Penedès, D. O. Priorat, y D. O. Montsant. Pasos. Revista de Turismo y Patrimonio Cultural, 6 (3), 493-509. (2008).

MILLÁN, M. G.; DANCAUSA M. G. El desarrollo turístico de zonas rurales en España a partir de la creación de rutas del vino: un análisis DAFO. Teoría y Praxis, (12), 5279. (2012a).

MILLÁN VÁZQUEZ DE LA TORRE, G.; MELIÁN NAVARRO, A. Rutas turísticas enológicas y desarrollo rural. El caso de estudio de la denominación de origen MontillaMoriles en la provincia de Córdoba. Papeles de Geografía, (47-48), 159-170. 2008.

MIRANDA ESCOLAR, B.; FERNÁNDEZ MORUECO, R. Vino, turismo e innovación: las Rutas del Vino de España, una estrategia integrada de desarrollo rural. Estudios de Economía Aplicada, 29 (1), 129-164. 2011.

PICTE - PLAN INTEGRAL DE CALIDAD DEL TURISMO 2000-2006. Centro de Publicaciones y Documentación del Ministerio de Economía y Acienda, Madrid, 1999. 
RAMIS HERNÁNDEZ, A. Turismo y Vino en el Mundo. El caso de bodegas Enrique Mendoza España. Apuntes de la Universidad de Alicante. Disponible en web: $<$ http://rua.ua.es/dspace/bitstream/10045/15168/1/Turismo\%20y\%20vino\%20en\%20el \%20mundo_Ramis.pdf. 2010>. Accedido en: 20/10/2014.

RECEVIN. RED EUROPEA DE CIUDADES DEL VINO. Carta Europea del Enoturismo. Disponible en: <http://www.recevin.net/downloads/Charte_ES.pdf>. 2006.

RODRIGUES GARCÍA, J.; LÓPEZ-GUZMAN, T.; SÁNCHEZ CAÑIZARES, S.; JIMÉNEZ GARCÍA, M. Turismo del vino en el Marco de Jerez. Un análisis desde la perspectiva de la oferta. Cuadernos de Turismo, (26), 217-334. 2010.

RUTAS DEL VINO DE ESPANA. ACEVIN. Disponible en: <http://www.wineroutesofspain.com/ver/2457/Rutas-del-vino.html>. 2012. Accedido en: $01 / 05 / 2013$.

SIMÕES, O. Enoturismo em Portugal: as rotas de vino. Pasos. Revista de Turismo y Patrimonio Cultural, 6 (2), 269-279. 2008.

TALLARICO, G. La construcción comunicativa de las denominaciones de origen. Una aproximación al análisis del sector vitivinícola español. Revista Latina de Comunicación Social, (34). Disponible en: <http://www.ull.es/publicaciones/latina/aa2000kjl/w34oc/47s4talla.htm>. 2000.

Accedido en: 18/09/2014.

TELFER, D. Strategic alliances along the Niagara Wine Route. Tourism Management, 22(1), 21-30. 2001.

TURGALICIA - GUÍA DE LAS RUTAS DE LOS VINOS DE GALICIA. Turgalicia. FEDER. Galicia, 2010.

XUNTA DE GALICIA. Rutas do viño Galicia Norte de Portugal. Disponible en: <http://rutasdelvino.xunta.es/es/. 2014>. Accedido en 20/10/2014.

Recebido em: 15-05-2014.

Aprovado em: 13-06-2014. 